\title{
Search for light scalar dark matter with atomic gravitational wave detectors
}

\author{
Asimina Arvanitaki, ${ }^{1, *}$ Peter W. Graham, ${ }^{2, \dagger}$ Jason M. Hogan, ${ }^{3, \star}$ Surjeet Rajendran, ${ }^{4, \S}$ and Ken Van Tilburg ${ }^{2,5,6, \|}$ \\ ${ }^{1}$ Perimeter Institute for Theoretical Physics, Waterloo, Ontario N2L 2Y5, Canada \\ ${ }^{2}$ Stanford Institute for Theoretical Physics, Stanford University, Stanford, California 94305, USA \\ ${ }^{3}$ Department of Physics, Stanford University, Stanford, California 94305, USA \\ ${ }^{4}$ Berkeley Center for Theoretical Physics, Department of Physics, University of California, \\ Berkeley, California 94720, USA \\ ${ }^{5}$ School of Natural Sciences, Institute for Advanced Study, Princeton, New Jersey 08540, USA \\ ${ }^{6}$ Center for Cosmology and Particle Physics, Department of Physics, New York University, \\ New York, New York 10003, USA
}

(Received 25 August 2016; published 16 April 2018)

\begin{abstract}
We show that gravitational wave detectors based on a type of atom interferometry are sensitive to ultralight scalar dark matter. Such dark matter can cause temporal oscillations in fundamental constants with a frequency set by the dark matter mass and amplitude determined by the local dark matter density. The result is a modulation of atomic transition energies. We point out a new time-domain signature of this effect in a type of gravitational wave detector that compares two spatially separated atom interferometers referenced by a common laser. Such a detector can improve on current searches for electron-mass or electric-charge modulus dark matter by up to 10 orders of magnitude in coupling, in a frequency band complementary to that of other proposals. It demonstrates that this class of atomic sensors is qualitatively different from other gravitational wave detectors, including those based on laser interferometry. By using atomic-clock-like interferometers, laser noise is mitigated with only a single baseline. These atomic sensors can thus detect scalar signals in addition to tensor signals.
\end{abstract}

DOI: 10.1103/PhysRevD.97.075020

\section{INTRODUCTION}

The search for dark matter (DM) is one of the most important goals in particle physics. There are now many experiments designed for the direct detection of DM. Almost all of these search for heavier DM, with mass well above an $\mathrm{eV}$, using energy deposition from DM particles scattering in the detector. Traditional particle detection techniques have energy thresholds which make it challenging to look for lighter DM. However, there is a vast range of DM parameter space with mass far below the level detectable in these experiments. New types of technology are required to search for ultralight DM.

The QCD axion is perhaps the best known example of light DM, but there are many other motivated possibilities

\footnotetext{
*aarvanitaki@perimeterinstitute.ca

†pwgraham@stanford.edu

thogan@stanford.edu

\$surjeet@berkeley.edu

"kenvt@ias.edu; kenvt@nyu.edu
}

Published by the American Physical Society under the terms of the Creative Commons Attribution 4.0 International license. Further distribution of this work must maintain attribution to the author(s) and the published article's title, journal citation, and DOI. Funded by SCOAP. such as light moduli [1-4], dilatons [5,6], Higgs portal DM [7], and the relaxion [8] among many others. We focus on DM with scalar couplings to matter, which causes time variation of fundamental constants such as the electron mass [9]. This type of DM can be searched for using atomic clocks [9-11], resonant-mass detectors [12], and accelerometers [13]. Interesting astrophysical signatures in pulsar systems can also arise for minimal [14] and nonminimal [15] couplings to gravity.

In this paper, we demonstrate that a class of atomic sensors for gravitational waves [16] can be used for direct detection of scalar DM over many orders of magnitude in mass. This type of atomic sensor is unique in that it has full sensitivity with a single baseline because it relies on atom interferometers designed to be similar to atomic clocks. As a single-baseline detector it does not rely on the tensor nature of the gravitational wave $(\mathrm{GW})$. This makes it ideal for searching for scalar DM as well.

\section{MODEL}

A scalar DM particle will naturally couple to the Standard Model particles, and hence potentially be observable, through a relatively small number of couplings in the effective field theory. In this paper, we consider a representative set of its couplings, described by the Lagrangian 


$$
\begin{aligned}
\mathcal{L}= & +\frac{1}{2} \partial_{\mu} \phi \partial^{\mu} \phi-\frac{1}{2} m_{\phi}^{2} \phi^{2} \\
& -\sqrt{4 \pi G_{N}} \phi\left[d_{m_{e}} m_{e} \bar{e} e-\frac{d_{e}}{4} F_{\mu \nu} F^{\mu \nu}\right],
\end{aligned}
$$

where we parametrized the leading interaction with electrons and photons relative to gravity as in Refs. [17,18]; $G_{N}$ is Newton's constant, so $d_{m_{e}}=d_{e}=1$ would be the couplings of a scalar graviton. We employ units in which $\hbar=c=1$. The couplings in Eq. (2) could originate from a Higgs portal coupling of the form $\mathcal{L} \supset b \phi|H|^{2}$, which is one ultraviolet completion into a renormalizable model with a particularly low cutoff [7]. Scalar fields with quadratic couplings to matter [19-21], e.g. $\mathcal{L} \supset \phi^{2}|H|^{2}$, give rise to analogous signatures as the linear couplings but have drastically more fine-tuned masses for the same physical effect, so we shall not consider them further.

Bosonic DM much lighter than $1 \mathrm{eV}$ is a highly classical state because of high occupation numbers and can be approximated by a nonrelativistic plane wave solution to Eq. (1),

$$
\phi(t, \mathbf{x})=\phi_{0} \cos \left[m_{\phi}(t-\mathbf{v} \cdot \mathbf{x})+\beta\right]+\mathcal{O}\left(|\mathbf{v}|^{2}\right),
$$

with amplitude $\phi_{0} \simeq \sqrt{2 \rho_{\mathrm{DM}}} / m_{\phi}$ determined by the local DM energy density $\rho_{\mathrm{DM}} \approx 0.3 \mathrm{GeV} / \mathrm{cm}^{3}$. The local description of Eq. (3) should be thought of as an incoherent superposition of waves (hence, $\phi_{0} \propto \sqrt{\rho_{\mathrm{DM}}}$ ) that nevertheless has a long phase coherence time of approximately $2 \pi / m_{\phi} v_{\text {vir }}^{2}$ where $v_{\text {vir }} \sim 10^{-3}$ is the Galactic virial velocity. The coherence arises from the nonrelativistic nature of DM: the angular frequency of the wave is mostly set by the rest-mass energy $m_{\phi}$. It receives small kinetic energy corrections of $\mathcal{O}\left(m_{\phi} v_{\text {vir }}^{2}\right)$, which do have a large spread: $\langle|\mathbf{v}|\rangle \sim v_{\text {vir }}$ and $\left\langle(\mathbf{v}-\langle\mathbf{v}\rangle)^{2}\right\rangle \sim v_{\text {vir }}^{2}$.

The scalar field DM oscillations of Eq. (3) combined with the couplings to matter of Eq. (2) cause fundamental "constants" such as the electron mass and the fine-structure constant to oscillate in time:

$$
\begin{gathered}
m_{e}(t, \mathbf{x})=m_{e}\left[1+d_{m_{e}} \sqrt{4 \pi G_{N}} \phi(t, \mathbf{x})\right] \\
\alpha(t, \mathbf{x})=\alpha\left[1+d_{e} \sqrt{4 \pi G_{N}} \phi(t, \mathbf{x})\right] .
\end{gathered}
$$

Temporal variation of $m_{e}$ and $\alpha$ gives rise to oscillations in energy and length scales in atoms, phenomena which were respectively exploited by DM search proposals using atomic-clock pairs [9] and resonant-mass detectors [12]. Spatial variation leads to oscillating, chemistry-dependent forces, which can be looked for with accelerometers [13] (see also Ref. [9] for a tidal-force effect).

We will show that the DM-induced temporal variation of atomic transition frequencies can be searched for with a single atomic species by exploiting the time-domain response of a differential atomic interferometer to a scalar DM wave. The search strategy outlined below has a discovery reach for scalar DM couplings that is potentially orders of magnitude better than existing constraints and proposals in its frequency band and is complementary to the low-frequency, broadband strategies of Refs. $[9,13]$ and the high-frequency, resonant searches of Ref. [12].

An electronic transition energy $\omega_{\mathrm{A}}$ depends on the values of $m_{e}$ and $\alpha$ and so will oscillate itself in the presence of a scalar DM wave:

$$
\begin{aligned}
\omega_{\mathrm{A}}(t) & \simeq \omega_{\mathrm{A}}+\Delta \omega_{\mathrm{A}} \cos \left(m_{\phi} t\right) ; \\
\Delta \omega_{\mathrm{A}} & \equiv \omega_{\mathrm{A}} \sqrt{4 \pi G_{N}} \phi_{0}\left(d_{m_{e}}+\xi d_{e}\right) .
\end{aligned}
$$

Above, we have neglected the $v_{\text {vir }}$-suppressed spatial variation and the $v_{\mathrm{vir}}^{2}$-suppressed temporal incoherence, which we will restore in our final results. We assumed a linear dependence of $\omega_{\mathrm{A}}$ on $m_{e}$, valid to a high degree for all (nonhyperfine) electronic transitions. For the $5 \mathrm{~s}^{2}{ }^{1} \mathrm{~S}_{0} \leftrightarrow$ $5 \mathrm{~s} 5 \mathrm{p}^{3} \mathrm{P}_{0}$ transition in $\mathrm{Sr} \mathrm{I}$, which we will take as a case study throughout, one has $\xi \approx 2.06$ [22]. The dependence of other atomic transition energies on fundamental constants may be found in Refs. [23-25].

\section{PHYSICAL EFFECT}

The light-pulse atom interferometry scheme of Ref. [16], depicted in Fig. 1, is like a differential atomic clock, where one laser is referenced to two spatially separated atomic ensembles. In the absence of new physics and reducible backgrounds, the phase response in the atomic ensembles is identical, but both suffer from laser noise imprinted onto the atoms, especially at frequencies below $1 \mathrm{~Hz}$. However, the differential atomic phase response is insensitive to laser noise when the atoms move along free-fall geodesics when not manipulated by the laser. This differential phase response can serve as a low-background channel to look for new physics. A GW would modulate the light travel time of the laser pulses between the atomic ensembles, leading to a differential phase accumulation over the interferometer sequence [16].

Atomic sensors for GW detection are also intrinsically sensitive to modulus DM waves, without change to the experimental configuration. Given that all phases in the sequence of Fig. 1 cancel in absence of new physics, we keep track only of DM-induced phase accumulation $\Phi$ of the excited atomic state relative to that of the ground state. [Effects on photon propagation from the $d_{e}$ coupling of Eq. (2) in a background scalar DM field can be ignored since it does not change the speed nor frequency of the laser light in vacuum.] Between times $t_{0}$ and $t_{1}$, this amounts to 


$$
\Phi_{t_{0}}^{t_{1}} \equiv \int_{t_{0}}^{t_{1}} d t \Delta \omega_{\mathrm{A}} \cos \left(m_{\phi} t+\beta\right) .
$$

The signal phase $\Phi_{\mathrm{s}}$, is the difference between the phase of an atom interferometer located at $x_{1} \simeq 0$ and that of one at $x_{2} \simeq L$. For the setup in Fig. 1, this is approximately equal to

$$
\Phi_{\mathrm{s}} \simeq \Phi_{T-(n-1) L}^{T+L}-\Phi_{0}^{n L}-\Phi_{2 T-(n-1) L}^{2 T+L}+\Phi_{T}^{T+n L},
$$

where $T$ is half the time between the two $\pi / 2$ beam splitter pulses, $L \simeq x_{2}-x_{1}$ is the light travel time between the two laser sources, and $n$ is the number of large-momentumtransfer (LMT) photon kicks each atom receives. In the limits of $m_{\phi} \rightarrow 0$ and $m_{\phi} \rightarrow \infty, \Phi_{\mathrm{s}}$ asymptotes to zero. However, a nontrivial signal phase response does occur when the period of the DM wave matches the total duration of the interferometric sequence, namely $2 \pi / m_{\phi} \sim 2 T$.

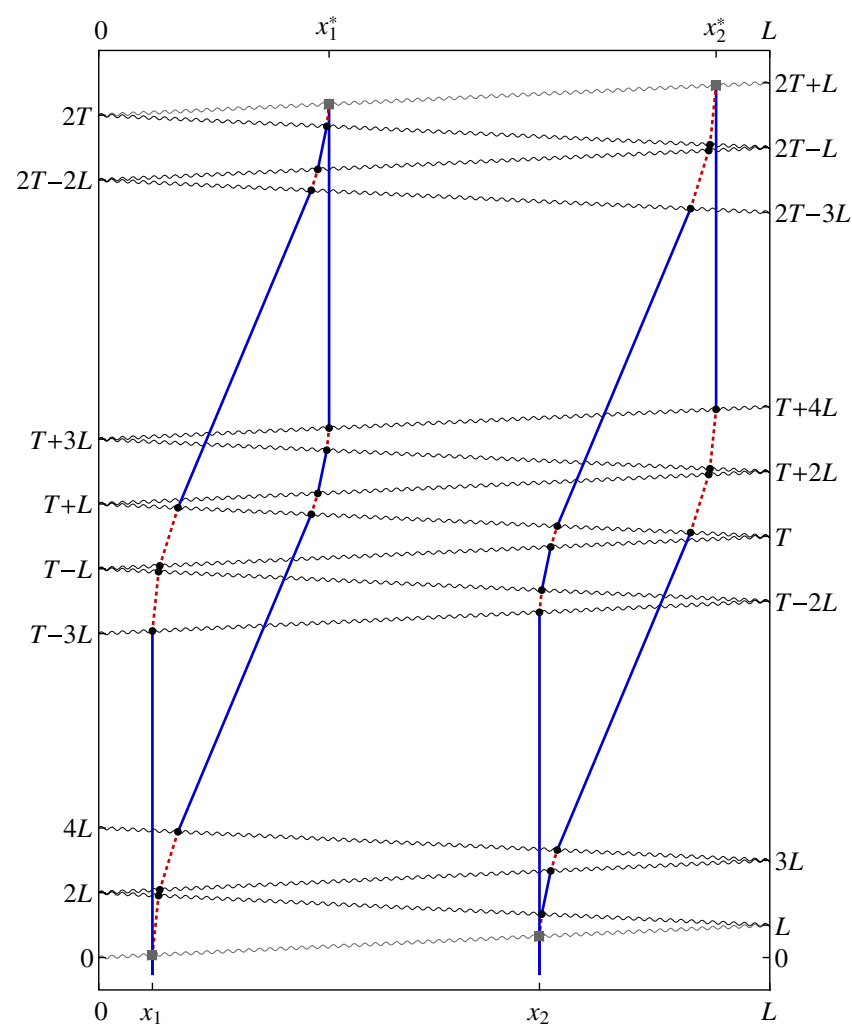

FIG. 1. Spacetime diagram of the light-pulse sequence on two atom interferometers, illustrated for $n=4$ (i.e., maximum four photon momenta transferred). A $\pi / 2$ laser pulse (gray, wavy) splits the wave function of atoms both at $x_{1}$ and at $x_{2}$ into the ground state $|g\rangle$ (blue, solid) and the excited state $|e\rangle$ (red, dashed) with equal probabilities. Subsequent $\pi$ laser pulses (black, wavy) exchange $|g\rangle \leftrightarrow|e\rangle$ and typically only interact with one branch of each wave function due to Doppler shifts. A final $\pi / 2$ pulse interferes the wave function of both interferometers. Interaction points are indicated by gray squares (black dots) for $\pi / 2(\pi)$ pulses. For clarity, atomic separations are exaggerated; realistically, $x_{1} \sim x_{1}^{*} \sim L-x_{2} \sim L-x_{2}^{*} \ll L$.
(By construction, $T>n L$, and $T \gg L$ for the setups under consideration.) For example, in the optimally matched case with a DM phase $\beta=0$ at the start of the interferometric sequence, all of the terms in Eq. (9) are negative, because terms 2 and 3 ( 1 and 4 ) are generated during positive (negative) antinodes of the DM wave, yielding a signal phase shift of order $\Phi_{s} \sim-4 \Delta \omega_{\mathrm{A}}(n L)$.

The signal amplitude of Eq. (9), $\bar{\Phi}_{\mathrm{s}} \equiv\left(2 \int_{0}^{2 \pi} d \beta \Phi_{\mathrm{s}}^{2} / 2 \pi\right)^{1 / 2}$, for general $m_{\phi}$ is

$$
\begin{aligned}
\bar{\Phi}_{\mathrm{s}}= & 8 \frac{\Delta \omega_{\mathrm{A}}}{m_{\phi}} \\
& \times\left|\sin \left[\frac{m_{\phi} n L}{2}\right] \sin \left[\frac{m_{\phi}(T-(n-1) L}{2}\right] \sin \left[\frac{m_{\phi} T}{2}\right]\right| .
\end{aligned}
$$

Since $\Delta \omega_{\mathrm{A}} \propto 1 / m_{\phi}$ at fixed DM energy density [see below Eq. (3)], we deduce that the effect decouples $\propto m_{\phi}$ for $m_{\phi} \rightarrow 0$ and $\propto 1 / m_{\phi}^{2}$ for $m_{\phi} \rightarrow \infty$. The zero-frequency limit above is consistent with the fact that in absence of a reference scale the variation of a dimensionful quantity such as $\Delta \omega_{\mathrm{A}}$ (and thus $m_{e}$ ) becomes unobservable. The phase shift $\Phi_{\mathrm{s}}$ is a dimensionless observable that is sensitive to fractional variation of the atomic transition energy over different parts of spacetime and constitutes a new, unique time-domain signature of light modulus dark matter.

The experiment under consideration can be thought of as a comparison of two atomic clocks. Here, the clocks are spatially separated, which is what makes it a GW detector. This also creates a difference in the effect of the scalar DM on the two clocks, allowing a differential measurement to cancel laser noise but not the DM signal. This observable effect differs from that of other proposed experiments searching for scalar DM using atomic-clock-based technology. For example, our proposal shows that the scalar DM effect can be detected using a single species of atoms, in contrast to the methods of Ref. [9]. This is because the differential setup of Ref. [16] allows a comparison of the response of two otherwise identical atomic clocks at different points in time, which is kept by the phase evolution of the DM wave.

The GW sensors of the type described in Ref. [16] have two key advantages over searches with collocated atomic clocks advocated in Ref. [9]. First, the differential measurement under consideration is less susceptible to laser frequency noise than a setup comparing two different atomic transition energies with two separate lasers. Second, our proposal exploits the time-domain response of the $\mathrm{GW}$ sensor to a DM wave rather than the equivalenceprinciple-violating nature of the DM couplings to atomic transition frequencies, providing prime sensitivity to the $d_{m_{e}}$ coupling without having to rely on comparatively less precise hyperfine clock transitions. The recent GW detector design 
of Ref. [26] likewise reaps the benefits of this second advantage since the same physical measurement is performed and would have the same sensitivity as our proposal, provided contributions from laser frequency noise can be mitigated to below atomic shot noise levels.

Additionally, our proposal differs from the recent Ref. [27], which also proposed atom interferometer GW detectors for scalar DM detection. We consider the direct effect of the scalar DM on the internal state of the atomic ensemble, while Ref. [27] mainly relies on the DM effect on the Earth's gravitational field. Our proposal achieves the best discovery potential in the most sensitive frequency band of GW detectors, while Ref. [27] is sensitive only to lower-frequency signals.

\section{EXPERIMENTAL SENSITIVITY}

The scheme proposed in Ref. [16] can be realized in a ground-based interferometer as well as in a space-based satellite antenna. A terrestrial experiment could be operated in a vertical shaft of length $L=10^{3} \mathrm{~m}$ with $10 \mathrm{~m}$ interferometers at the top and bottom, allowing free-fall times of $T=1.4 \mathrm{~s}$. We restrict to a maximum number of $N_{\max }=10^{3}$ laser pulses in order to retain atom number, which in turn limits the number of LMT kicks to $n=250$. We assume shot-noise-limited sensitivity above $f=10^{-1} \mathrm{~Hz}$ with a noise spectral density $\sqrt{S_{\Phi}} \approx 10^{-5} / \mathrm{Hz}^{1 / 2}$, made possible with an atomic flux of $10^{10} / \mathrm{s}$, or with fewer atoms and significant squeezing [28].

A space-based satellite experiment can exhibit a much longer baseline length $L$ and interrogation time $T$, because the laser platforms can move on free-fall geodesics along with the atoms. A GW antenna design using atom interferometry near satellites connected with heterodyne laser links [29] has a proposed configuration with $L=6 \times 10^{8} \mathrm{~m}$, $T=160 \mathrm{~s}$, and $n=12$. The baseline length and interrogation time are limited by laser diffraction and atomic loss due to scattering with background gas and light, respectively. For this setup, we assume a shot-noise-limited sensitivity of $\sqrt{S_{\Phi}} \approx 10^{-4} / \mathrm{Hz}^{1 / 2}$.

Given a DM signal bandwidth of $\Delta f_{\phi} \simeq m_{\phi} v_{\mathrm{vir}}^{2} / 2 \pi$, differential atomic phase oscillations with square amplitude as small as $\delta \Phi_{\mathrm{s}}^{2}=S_{\Phi} t_{\text {int }}^{-1} \max \left\{1, t_{\text {int }} \Delta f_{\phi}\right\}^{1 / 2}$ may be detected at unit signal-to-noise ratio $(\mathrm{SNR}=1)$ after an integration time $t_{\text {int }}=10^{8} \mathrm{~s}$. With the parameters for $L, T$, and $n$, and the atomic transition (throughout assumed to be the ${ }^{1} S_{0} \leftrightarrow$ ${ }^{3} \mathrm{P}_{0}$ transition in $\mathrm{Sr} \mathrm{I}$ ), the discovery reach for DM couplings can then be computed as a function $m_{\phi}$ with the aid of Eqs. (7) and (10).

Atomic sensors provide extraordinary discovery reach, with a potential to improve on existing constraints and other proposals by many orders of magnitude over a wide frequency band. In Fig. 2, we plot the sensitivity to the electron coupling $d_{m_{e}}$ (top panel) and photon coupling $d_{e}$ (bottom panel) for both the terrestrial ("AI-TB," light blue)

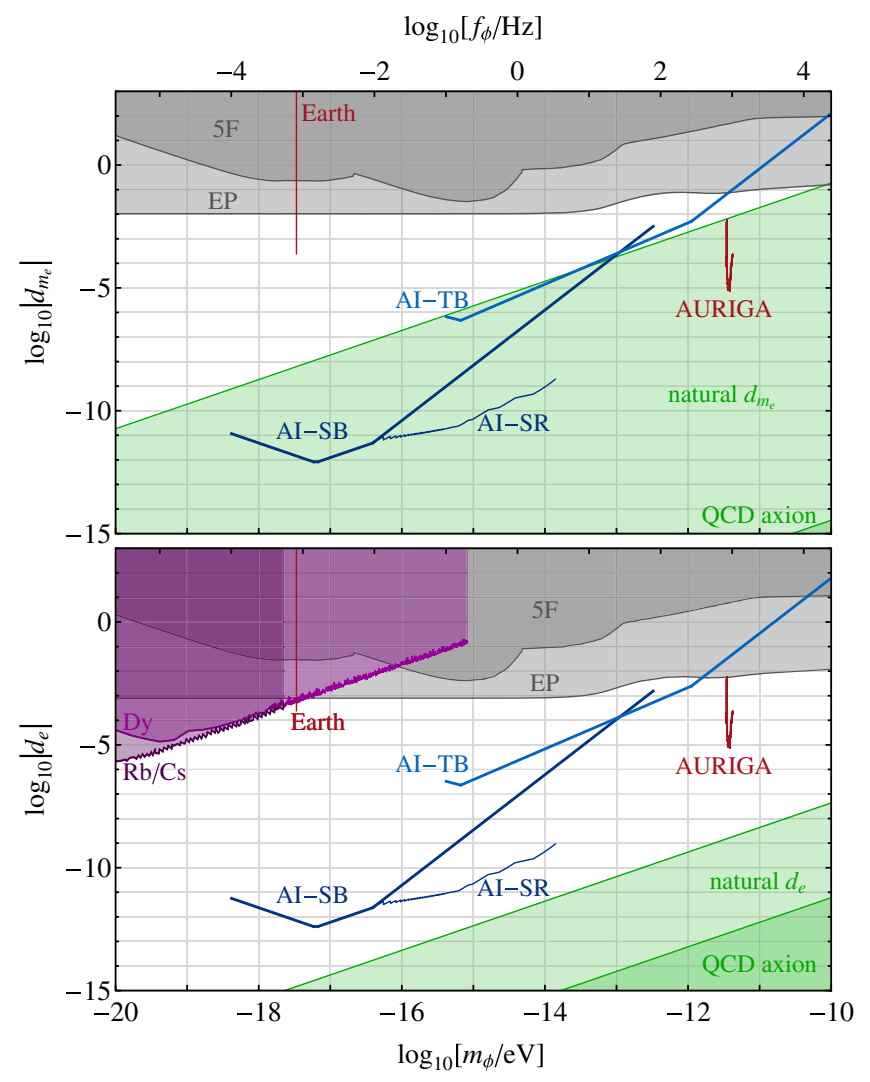

FIG. 2. Parameter space for the coupling $d_{m_{e}}$ to electrons (top panel) and $d_{e}$ to photons (bottom panel), as a function of dark matter mass $m_{\phi}$. Blue curves depict the SNR $=1$ sensitivity envelopes of the proposed atomic sensors: a terrestrial experiment operated in broadband mode ("AI-TB"); a long-baseline, broadband, space-based antenna ("AI-SB"); and a shorter, resonant satellite antenna ("AI-SR"). Also depicted are 95\% C.L. constraints from searches for new Yukawa forces that violate/ conserve the equivalence principle ("EP/5F", gray regions) [32-34], atomic spectroscopy data in Dy and in Rb/Cs (light and dark purple regions) $[10,11]$, seismic data on the fundamental breathing mode of Earth (red) [35], and a search for acoustic excitations in the AURIGA resonant-mass detector (red band) [36]. Green regions show natural parameter space for a $10-\mathrm{TeV}$ cutoff, and allowed parameter space for the QCD axion.

and space-based proposals ("AI-SB," dark blue). Analogous curves for the Higgs portal coupling are plotted in Fig. 3. For clarity, we used the approximation $|\sin (x)| \sim \min \{x, 1 / \sqrt{2}\}$ for the power-averaged envelope of Eq. (10). We note that a DM signal with frequency above the repetition frequency of the interferometer sequence (typically about $1 \mathrm{~Hz}$ ) will be aliased to lower frequencies and can still be detected with the same phase sensitivity over the parameter space of interest. We show the reach of the space-based proposal up to frequencies where the DM wave becomes spatially incoherent (when $m_{\phi} v_{\text {vir }} L \gtrsim 1$ ) and down to frequencies where gravity gradients are deemed to become more important than shot noise, at $f \lesssim 10^{-4} \mathrm{~Hz}$. For the ground-based proposal, 


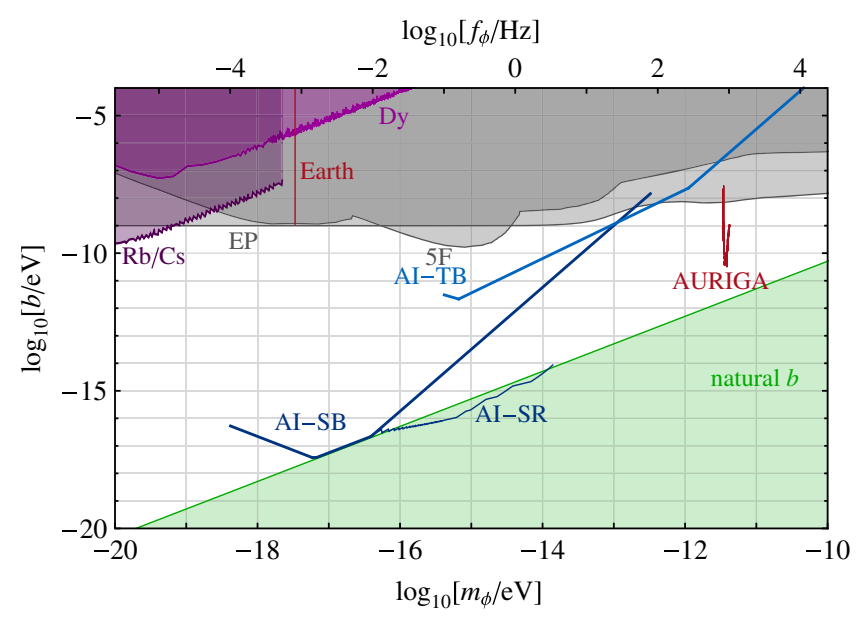

FIG. 3. Parameter space for the Higgs portal coupling $b$ as a function of dark matter mass $m_{\phi}$. Curves and regions are as in Fig. 2. Here, the green region highlights couplings $b$ for which the lightest physical mass eigenvalue $m_{\phi}$ of the scalar potential is natural at the classical level, as described in the text. Loop-level quantum corrections to the mass are subdominant, so the natural region is independent of the UV cutoff.

gravity gradients can likely be kept subdominant for $f \gtrsim 10^{-1} \mathrm{~Hz}[30,31]$.

In Figs. 2 and 3, we also show 95\% C.L. gray exclusion regions from equivalence-principle tests [32,33] and searches for a Yukawa-type deviation from the gravitational force [34], which are both independent of DM abundance. Atomic spectroscopy limits at $95 \%$ C.L. on oscillations of relative transition energies in isotopes of Dy [10] and in $\mathrm{Rb}$ and Cs [11] are also shown. In red, we plot limits from atomic length scale oscillation effects [12]: one derived from terrestrial seismic data [35] and another from a search for monochromatic scalar strain signals [36] in the AURIGA resonant-mass detector [37]. Reinterpretations in terms of constraints on $b$ were first presented in Ref. [7] for force tests, in Ref. [13] for Dy spectroscopy, and in Ref. [38] for Rb/Cs spectroscopy.

Green regions in Fig. 2 indicate natural parameter spacewhere loop-level quantum corrections to the scalar mass are less than the physical mass $m_{\phi}$ for an ultraviolet cutoff of $10 \mathrm{TeV}$ - as well as allowed parameter space for the QCD axion. In Fig. 3, the green region highlights natural Higgs portal couplings - regardless of the UV cutoff. Elsewhere, the coupling $b$ is tuned against the bare mass of $\phi$ at the classical level (loop corrections are subdominant), such that the lightest mass eigenstate in the $\phi-H$ scalar potential has a physical mass $m_{\phi} \lesssim b / \sqrt{2 \lambda}$. This mass eigenstate is rotated slightly in the Higgs direction, by an angle $b / \sqrt{2 \lambda m_{h}^{2}}$ with $m_{h}$ the heavy mass eigenvalue at $125 \mathrm{GeV}$ and the Higgs quartic normalized as $\mathcal{L} \supset \lambda|H|^{4}$, leading to, e.g., the correspondence $d_{m_{e}} \sqrt{4 \pi G_{N}} \simeq b / m_{h}^{2}[7,13]$.

The atomic sensors of the type described in Ref. [16] are broadband sensors, but they can also be operated in resonant mode. By interweaving many diamond-shaped atomic paths of the type in Fig. 1 in a fixed interferometer duration, the detectors become resonantly sensitive to higherfrequency signals [39]. With a $Q_{d}$ number of diamonds in a total sequence duration $\tilde{T}$, maximum sensitivity is achieved at $f_{d} \sim Q_{d} / \tilde{T}$ (and integer multiples thereof) in a frequency band $\Delta f_{d} \sim f_{d} / Q_{d}$. Constrained on keeping the total time below $T_{\max }=300 \mathrm{~s}$ and the total number of laser pulses below $N_{\max }=10^{3}$, a strain spectral noise density of $\sqrt{S_{\mathrm{h}}} \sim$ $10^{-22} / \mathrm{Hz}^{1 / 2}$ may be attained with a baseline of $L=4.4 \times$ $10^{7} \mathrm{~m}$ and phase noise $\sqrt{S_{\Phi}} \approx 10^{-5} / \mathrm{Hz}^{1 / 2}$ in a frequency range between $f_{\min }=0.01 \mathrm{~Hz}$ and $f_{\max }=4 \mathrm{~Hz}$ [39].

Sweeping through this band with a constant average fractional frequency scanning speed of $\sigma \equiv \Delta f / f \Delta t$ by changing $Q_{d}$ and $\tilde{T}$ would take an integration time of $t_{\text {int }}=\sigma^{-1} \ln \left(f_{\max } / f_{\min }\right)$, again taken to be $10^{8} \mathrm{~s}$. This yields a reach for, e.g., $d_{m_{e}}$ of approximately $\sqrt{S_{\mathrm{h}} \sigma f / f_{\min }} / 2 \sqrt{4 \pi G_{N}} \phi_{0}$, which is plotted as the thin blue curve ("AI-SR") in the top panel Fig. 2, with analogous results for $d_{e}$ and $b$ in Figs. 2 and 3. The resonant mode would allow precise dissection of a positive narrow band signal. It can also be realized in the above terrestrial detector, though with fewer sensitivity benefits of a scanning search relative to broadband operation.

As evident from Figs. 2 and 3, atomic GW detectors provide excellent discovery potential complementary to that of other detection strategies for light moduli. Our proposed searches can potentially reach couplings up to 10 orders of magnitude weaker than current EP and fifth-force limits if $\phi$ constitutes all of the DM; conversely, they can probe new parameter space even if $\phi$ makes up only a minuscule $10^{-20}$ fraction of the DM energy density. The near-complete elimination of laser frequency noise and comparatively long interrogation time of atoms in free fall means that our proposal can likely improve on searches using different but collocated atomic clocks [9] at frequencies above $10^{-4} \mathrm{~Hz}$. Collocated atomic-clock comparisons exhibit better sensitivity at lower frequencies and have much room for improvement, especially with the increasing performance of optical lattice clocks [40-44] and the future development of a nuclear clock in ${ }^{229} \mathrm{Th}$ [45-48]. Our proposed setup also complements scalar DM searches with resonant-mass detectors [12], which are more sensitive at higher frequencies but severely limited by vibration and seismic noise below $100 \mathrm{~Hz}$.

Atomic GW detectors also have significant sensitivity to topological defects of fields with scalar couplings [49], through two separate physical effects, both yielding transient signals. The first arises when one atom interferometer is inside a defect and thus has its atomic transition energies shifted relative to those of the other interferometer. The second effect results from the field gradient at the defect edges, producing differential forces $[9,13]$ and thus apparent strains on the interferometers. The second signature is 
present in any $\mathrm{GW}$ detector with free-falling test masses, including aLIGO [50]. Our preliminary estimates show that $\mathrm{GW}$ detectors have a potential sensitivity much beyond that of atomic-clock experiments [49,51] and astrophysical observations [52], warranting a detailed analysis elsewhere.

\section{CONCLUSION}

Most GW detection techniques based on interferometry rely on the tensor nature of the GW and have significantly reduced sensitivity to scalar signals, including those from scalar DM. Laser interferometers such as aLIGO [50] or the proposed eLISA [53] and atom interferometers such as AGIS $[30,31,54]$ compare the signal in multiple directions in order to cancel out laser noise, which would otherwise severely limit the sensitivity. Laser frequency noise is similar to the scalar DM effect, since it acts on both baselines in the same way. By looking for a differential response on multiple equal-length baselines, these GW detectors drastically reduce any signal from scalar DM.

However, the GW detector described in Ref. [16] uses a differential measurement of two atom interferometers designed to be similar to atomic clocks. The use of atomic-clock-like interferometers allows the removal of laser noise along a single baseline, unlike in many other GW detectors. It is ideally suited for a scalar DM search, which can be run simultaneously and parasitically alongside a
GW search using the same detector readout strategy. In this paper, we identified a new signature of scalar dark matter in an atomic sensor of this type and outlined search strategies in a wide range of natural parameter space for well-motivated dark matter candidates.

\section{ACKNOWLEDGMENTS}

We thank James Thompson and Jun Ye for helpful discussions. A. A. acknowledges the support of NSERC and the Stavros Niarchos Foundation. Research at Perimeter Institute is supported by the Government of Canada through Industry Canada and by the Province of Ontario through the Ministry of Economic Development and Innovation. P.W.G. acknowledges the support of NSF Grant No. PHY-1316706, DOE Early Career Award No. DE-SC0012012, and the W. M. Keck Foundation. S. R. was supported in part by the NSF under Grants No. PHY1417295 and No. PHY-1507160 and the Simons Foundation Grant No. 378243. This work was supported in part by the Heising-Simons Foundation Grants No. 2015-037 and No. 2015-038. K. V. T. was supported in part by the NSF under Grants No. PHY-1316753 and No. PHY-1620727, by a Schmidt Fellowship funded by the generosity of Eric and Wendy Schmidt, and also by financial support from the AMIAS.
[1] S. Dimopoulos and G. F. Giudice, Phys. Lett. B 379, 105 (1996).

[2] N. Arkani-Hamed, L. J. Hall, D. Smith, and N. Weiner, Phys. Rev. D 62, 105002 (2000).

[3] C. P. Burgess, A. Maharana, and F. Quevedo, J. High Energy Phys. 05 (2011) 010.

[4] M. Cicoli, C. P. Burgess, and F. Quevedo, J. High Energy Phys. 10 (2011) 119.

[5] T. Damour and A. M. Polyakov, Nucl. Phys. B423, 532 (1994).

[6] T. Taylor and G. Veneziano, Phys. Lett. B 213, 450 (1988).

[7] F. Piazza and M. Pospelov, Phys. Rev. D 82, 043533 (2010).

[8] P. W. Graham, D. E. Kaplan, and S. Rajendran, Phys. Rev. Lett. 115, 221801 (2015).

[9] A. Arvanitaki, J. Huang, and K. Van Tilburg, Phys. Rev. D 91, 015015 (2015).

[10] K. Van Tilburg, N. Leefer, L. Bougas, and D. Budker, Phys. Rev. Lett. 115, 011802 (2015).

[11] A. Hees, J. Gena, M. Abgrall, S. Bize, and P. Wolf, Phys. Rev. Lett. 117, 061301 (2016).

[12] A. Arvanitaki, S. Dimopoulos, and K. Van Tilburg, Phys. Rev. Lett. 116, 031102 (2016).

[13] P. W. Graham, D. E. Kaplan, J. Mardon, S. Rajendran, and W. A. Terrano, Phys. Rev. D 93, 075029 (2016).
[14] A. Khmelnitsky and V. Rubakov, J. Cosmol. Astropart. Phys. 02 (2014) 019.

[15] D. Blas, D. L. Nacir, and S. Sibiryakov, Phys. Rev. Lett. 118, 261102 (2017).

[16] P. W. Graham, J. M. Hogan, M. A. Kasevich, and S. Rajendran, Phys. Rev. Lett. 110, 171102 (2013).

[17] T. Damour and J. F. Donoghue, Classical Quantum Gravity 27, 202001 (2010).

[18] T. Damour and J. F. Donoghue, Phys. Rev. D 82, 084033 (2010).

[19] Y. V. Stadnik and V. V. Flambaum, Phys. Rev. Lett. 114, 161301 (2015).

[20] Y. V. Stadnik and V. V. Flambaum, Phys. Rev. Lett. 115, 201301 (2015).

[21] Y. V. Stadnik and V. V. Flambaum, Phys. Rev. A 93, 063630 (2016).

[22] E. J. Angstmann, V. A. Dzuba, and V. V. Flambaum, Phys. Rev. A 70, 014102 (2004).

[23] V. Dzuba, V. Flambaum, and J. Webb, Phys. Rev. A 59, 230 (1999).

[24] V. Dzuba, V. Flambaum, and J. Webb, Phys. Rev. Lett. 82, 888 (1999).

[25] V. Flambaum, Phys. Rev. Lett. 97, 092502 (2006).

[26] S. Kolkowitz, I. Pikovski, N. Langellier, M. D. Lukin, R. L. Walsworth, and J. Ye, Phys. Rev. D 94, 124043 (2016). 
[27] A. A. Geraci and A. Derevianko, Phys. Rev. Lett. 117, 261301 (2016).

[28] O. Hosten, N. J. Engelsen, R. Krishnakumar, and M. A. Kasevich, Nature (London) 529, 505 (2016).

[29] J. M. Hogan and M. A. Kasevich, Phys. Rev. A 94, 033632 (2016).

[30] S. Dimopoulos, P. W. Graham, J. M. Hogan, M. A. Kasevich, and S. Rajendran, Phys. Lett. B 678, 37 (2009).

[31] S. Dimopoulos, P. W. Graham, J. M. Hogan, M. A. Kasevich, and S. Rajendran, Phys. Rev. D 78, 122002 (2008).

[32] S. Schlamminger, K.-Y. Choi, T. A. Wagner, J. H. Gundlach, and E. G. Adelberger, Phys. Rev. Lett. 100, 041101 (2008).

[33] T. A. Wagner, S. Schlamminger, J. H. Gundlach, and E. G. Adelberger, Classical Quantum Gravity 29, 184002 (2012).

[34] E. Adelberger, B. R. Heckel, and A. Nelson, Annu. Rev. Nucl. Part. Sci. 53, 77 (2003).

[35] R. Weiss and B. Block, J. Geophys. Res. 70, 5615 (1965).

[36] A. Branca et al., Phys. Rev. Lett. 118, 021302 (2017).

[37] L. Baggio et al., Phys. Rev. Lett. 94, 241101 (2005).

[38] Y. V. Stadnik and V. V. Flambaum, Phys. Rev. A 94, 022111 (2016).

[39] P. W. Graham, J. M. Hogan, M. A. Kasevich, and S. Rajendran, Phys. Rev. D 94, 104022 (2016).

[40] N. Hinkley, J. A. Sherman, N. B. Phillips, M. Schioppo, N. D. Lemke, K. Beloy, M. Pizzocaro, C. W. Oates, and A. D. Ludlow, Science 341, 1215 (2013).
[41] T. L. Nicholson et al., Nat. Commun. 6, 6896 (2015).

[42] N. Nemitz, T. Ohkubo, M. Takamoto, I. Ushijima, M. Das, N. Ohmae, and H. Katori, Nat. Photonics 10, 258 (2016).

[43] N. Huntemann, C. Sanner, B. Lipphardt, C. Tamm, and E. Peik, Phys. Rev. Lett. 116, 063001 (2016).

[44] M. Schioppo et al., Nat. Photonics 11, 48 (2017).

[45] E. Peik and C. Tamm, Europhys. Lett. 61, 181 (2003).

[46] C. J. Campbell, A. G. Radnaev, A. Kuzmich, V. A. Dzuba, V. V. Flambaum, and A. Derevianko, Phys. Rev. Lett. 108, 120802 (2012).

[47] E. Tkalya, C. Schneider, J. Jeet, and E. R. Hudson, Phys. Rev. C 92, 054324 (2015).

[48] L. von der Wense et al., Nature (London) 533, 47 (2016).

[49] A. Derevianko and M. Pospelov, Nat. Phys. 10, 933 (2014).

[50] T. L. S. Collaboration, Classical Quantum Gravity 32, 074001 (2015).

[51] P. Wcislo, P. Morzynski, M. Bober, A. Cygan, D. Lisak, R. Ciurylo, and M. Zawada, arXiv:1605.05763.

[52] Y. V. Stadnik and V. V. Flambaum, Phys. Rev. Lett. 113, 151301 (2014).

[53] P. Amaro-Seoane et al., Low-frequency gravitational-wave science with eLISA/NGO, Technical Report, 2012.

[54] J. M. Hogan et al., Gen. Relativ. Gravit. 43, 1953 (2011). 